\title{
Twin relationship in between the variant-pair of $\eta$ precipitates in the Al-Zn-Mg-Cu aluminium alloy
}

\author{
Tsai-Fu Chung ${ }^{l}$, Yo-Lun Yang ${ }^{2}$, Chien-Nan Hsiao ${ }^{3}$, Wei-Chih Li ${ }^{4}$, Jer-Ren Yang ${ }^{l, *}$ \\ ${ }^{1}$ Department of Materials Science and Engineering, National Taiwan University, Taipei, Taiwan. \\ 2 Department of Mechanical Engineering, Imperial College London, London SW7 2AZ, UK. \\ ${ }^{3}$ Taiwan Instrument Research Institute, Hsinchu, Taiwan. \\ ${ }^{4}$ E.A. Fischione Instruments, Inc., 9003 Corporate Circle, Export, PA 15632, U.S.A.
}

\begin{abstract}
For Al- $\mathrm{Zn}-\mathrm{Mg}-\mathrm{Cu}$ aluminium alloys, 15 types of $\eta$ precipitates would possess the symmetrically variants distributed on the closed planes of the $\mathrm{Al}$ matrix, parallel to the $(0001) \eta,(2 \overline{1} \overline{1}) \eta$, or $(10 \overline{1} 0) \eta$ interfaces of precipitates. The $\eta_{2}$ precipitates, possessing the crystallographic orientation of $(1 \overline{1} \overline{1})_{\mathrm{Al}} / /(0001) \eta_{2}$ and $[110]_{\mathrm{Al}} / /[10 \overline{1} 0] \eta_{2}$, would exhibit four equivalent variants, i.e., $\eta_{2}{ }^{(1)}$ to $\eta_{2}{ }^{(4)}$, on the $(1 \overline{1} \overline{1})_{\mathrm{Al}}$ habit planes. In the present work, along the zone axis of $[110]_{\mathrm{Al}} / /[10 \overline{1} 0] \eta$, the edge-on configurations showing the twin-like atomic arrays would occur as the growth/coalescence of two $\eta_{2}$ variants grown on $(1 \overline{1} \overline{1})_{\mathrm{Al}}$ and $(1 \overline{1} 1)_{\mathrm{Al}}$ planes, respectively. The twin relationship can be revealed in term of the crystallographic relationship at $70.5^{\circ}$ with respect to their habit planes. Alternatively, on the $(\overline{1} \overline{1} 0)_{\mathrm{Al}}$ image, it also indicates the nearly-twinning configuration in between the variantpair of $\eta_{2}$.
\end{abstract}

\section{Introduction}

The Al-Zn-Mg-Cu aluminium alloys, exhibiting the high strength mechanical property, are well known to be applied in the automotive and aerospace applications. The precipitation hardening of the AA7050 aluminium alloys [1-9] begins from the fully or nearly coherent GP zones (GPI and GPII zones), and subsequently transforms into the semi-coherent $\eta^{\prime}$ precipitates (4 variants), and then the incoherent $\eta$ precipitates ( 15 types, $\eta_{1}-\eta_{14}$, and $\left.\eta_{4}{ }^{\prime}\right)$. In the recent work, The nucleation and transformation mechanisms of GPII zones $\rightarrow \eta^{\prime}$ precipitates and $\eta^{\prime}$ precipitates $\rightarrow \eta_{2}$ precipitates have been recognized as the separated and in-situ nucleations, which are conducted by the in-situ frames of micrographs and the serial FastFourier-Transform (FFT) diffractograms of highresolution transmission electron microscopy (HRTEM), respectively [1].

According to the in-situ transformation mechanism of $\eta^{\prime}$ precipitates $\rightarrow \eta_{2}$ precipitates and their identical crystallographic orientations with respect to the Al matrix : $[10 \overline{1} 0] \eta^{\prime} / /[110]_{\mathrm{Al}}$ and $(0001) \eta^{\prime} / /(1 \overline{1} \overline{1})_{\mathrm{Al}}$ of $\eta^{\prime}$ precipitates, and $[10 \overline{1} 0] \eta_{2} / /[110]_{\mathrm{Al}}$ and $(0001) \eta_{2} / /(1 \overline{1} \overline{1})_{\mathrm{Al}}$ of $\eta_{2}$ precipitates $[1,10]$, it would be observed four equivalent variants of $\eta_{2}$ (i.e., $\eta_{2}{ }^{(1)}$ precipitates to $\eta_{2}{ }^{(4)}$ precipitates), grown on the respective $(1 \overline{1} \overline{1})_{\mathrm{Al}}$ habit planes, as portrayed in Fig. 1. Afterwards, employed by Cs-corrected highangle annular-dark-field (HAADF) scanningtransmission electron microscopy (STEM) [2], the edgeon configurations of four $\eta_{2}$ along the zone axis of $[10 \overline{1} 0] \eta_{2} / /[110]_{\mathrm{Al}}$ are characterized as the sandwiched stacking arrangements, in which the regular variations of $\mathrm{Zn}$ and $\mathrm{Mg}$ layers subsequently stacks on the $(0001) \eta_{2} / /$ $(1 \overline{1} \overline{1})_{\mathrm{Al}}$ habit planes.

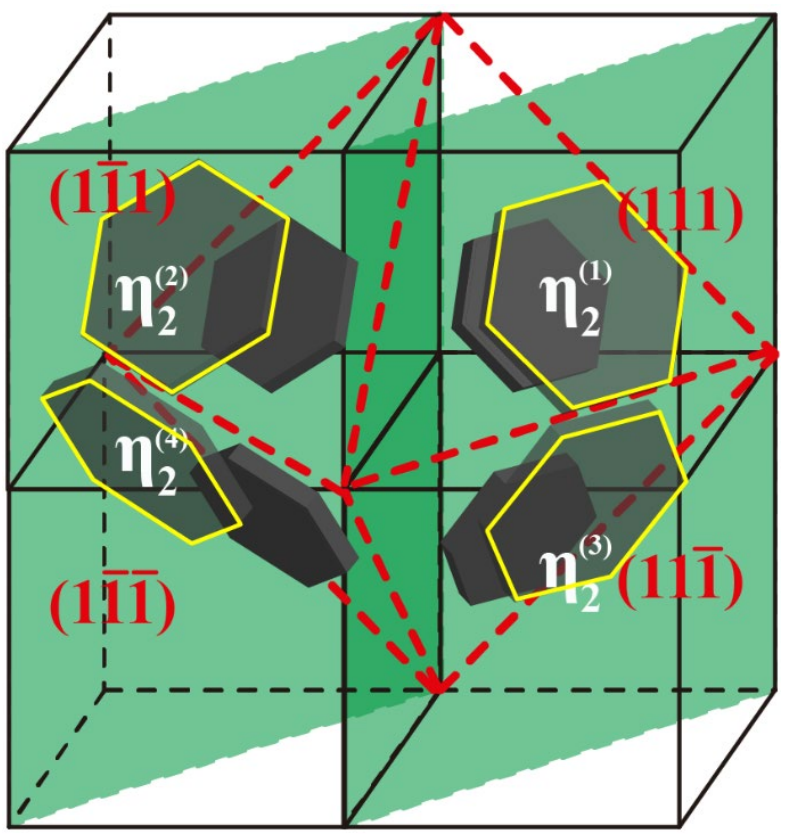

Fig. 1 The schematic diagram of four variants of $\eta_{2}$ precipitates (i.e., $\eta_{2}{ }^{(1)}$ to $\eta_{2}{ }^{(4)}$ ) grow on $\{111\}_{\text {Al }}$ habit planes. As the precipitate growing, the coarsened $\eta_{2}$ are indicated by yellow frames.

It has been reported that the distributions in between the orientational variants of precipitates would be easily misunderstood from the 2-dimensional projections of the 3 -dimensional phenomena $[11,12]$. For example, in the $\mathrm{Al}-\mathrm{Cu}-(\mathrm{Li})$ aluminium alloys [11], the impingements are inevitably caused by the overlap of two edge-on variants of $\theta^{\prime}-\mathrm{Al}_{2} \mathrm{Cu}$ precipitates, in which their habit planes are parallel to the $(100)_{\mathrm{Al}}$ and $(010)_{\mathrm{Al}}$ planes of the Al matrix, respectively. On the other hand, in the recent work employed by HAADF-STEM [12], the joint of two edgeon variants of $\theta^{\prime}$ incisively revealed the distributed

\footnotetext{
* Corresponding author: author@e-mail.org
} 
arrangements, implying that the possibility of the interaction between the orientational variants of precipitates. However, so far there is no direct experimental to support the crystallographic relationship such as twinning relationship in between two variants of $\theta^{\prime}$. Tacitly, in the $\mathrm{Al}-\mathrm{Zn}-\mathrm{Mg}-\mathrm{Cu}$ aluminium alloys, whether the crystallographic distribution of the 15 typed $\eta$ precipitates (i.e., $\eta_{1}-\eta_{14}$, and $\eta_{4}{ }^{\prime}$ ) and their corresponding variants $[1,2]$ would also exist the crystallographic relationship in between themselves has yet to be elucidated.

In the present work, employed by Cs-corrected HAADF STEM, we especially focused on the crystallographic relationships of $\eta_{2}$ and their variant pairs in $\mathrm{Al}-\mathrm{Zn}-\mathrm{Mg}-\mathrm{Cu}$ aluminium alloys. Along the zone axis of $[110]_{\mathrm{Al}}$, the detailed atomic configurations of $\eta_{2}$ has been revealed. Additionally, the crystallographic relationship in between variant-pair of $\eta_{2}$ has been confirmed under the observation of $[110]_{\mathrm{Al}}$ and $[\overline{1} \overline{1} 0]_{\mathrm{A} 1}$ zone axes

\section{Materials and methods}

The AA7050 aluminium alloy (Al-6.25Zn-2.14Mg$2.23 \mathrm{Cu}$, wt.\%) was used in this study. After solution treatment (at $475{ }^{\circ} \mathrm{C}$ for $1 \mathrm{~h}$ with water quenching), the samples were treated with a three-step ageing treatment: (1) ageing at $120^{\circ} \mathrm{C}$ for $8 \mathrm{~h},(2)$ ageing at $165^{\circ} \mathrm{C}$ for $1 \mathrm{~h}$, and subsequently (3) ageing at $174{ }^{\circ} \mathrm{C}$ for $8 \mathrm{~h}$ with an applied constant tensile stress of $162.5 \mathrm{MPa}$ at the same time (named as creep-age forming samples, CAF samples). The TEM specimens were prepared by cutting discs from the selected samples for observing the pancaked structures and thinning the discs to the thickness of $65-70 \mu \mathrm{m}$ before they were twin-jet electropolished in a mixture of $0.33 \mathrm{HNO}_{3}$ and $0.67 \mathrm{CH}_{3} \mathrm{OH}$ at $-20{ }^{\circ} \mathrm{C}$ with a working voltage of 10-11 V, as shown in Fig. 2.
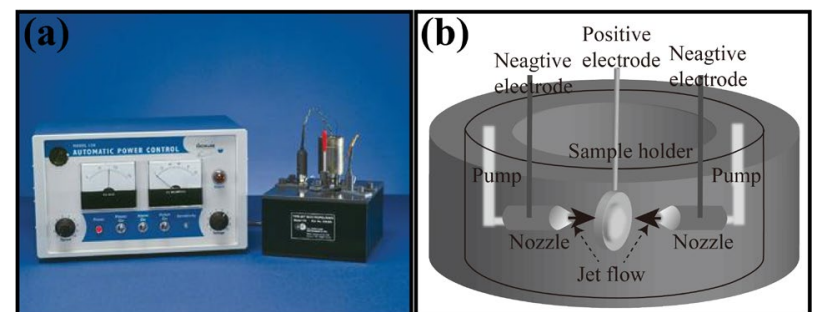

Fig. 2 (a) The twin-jet electropolishing instrument (E.A. Fischione Instrument), and (b) the schematic inward of twin-jet system.

The oxidation layers of specimens was removed and the corresponding thickness was reduced with an M1040 NanoMill system (E.A. Fischione Instrument), as shown in Fig. $3 \mathrm{a}$ and then the hydrocarbon contamination was cleaned by an M1070 Nanoclean plasma (E.A. Fischione Instrument), as shown in Fig. 3b. Afterwards, the TEM specimens were observed with an FEI Titan ChemiSTEM equipped with a spherical aberration corrector (Cscorrector), under the $135 \mathrm{~nm}$ camera length and with a collecting angle range of $\sim 8.24 \mathrm{mrad}$ (inner angle) to $\sim 143.6 \mathrm{mrad}$ (outer angle) of a HAADF detector.

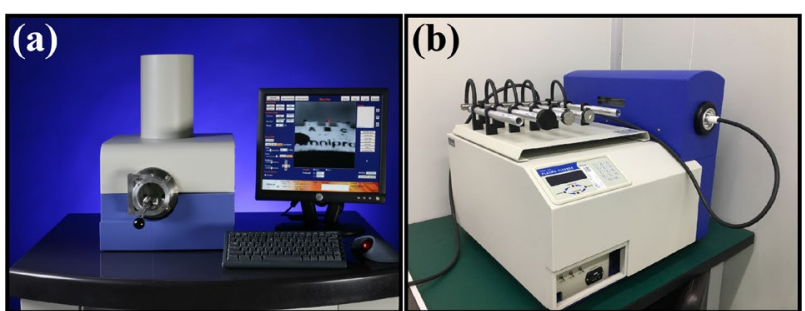

Fig. 3 (a) M1040 NanoMill system, and (b) M1070 Nanoclean plasma system.

\section{Results \& Discussion}

Fig. 4a shows the edge-on configuration of $\eta_{2}$ grown on the $(\overline{1} 11)_{\mathrm{Al}}$ habit plane, and its atomic arrangement along the $[110]_{\mathrm{Al}}$ zone axis. The alternative brightnessdarkness Z-contrast can be explained by the stimulated atomic image based on first principle calculation, as shown in Fig. 4b. The interweaving $\mathrm{Zn}$ and $\mathrm{Mg}$ atomiclayers are consistent with the brighter Z-contrast representing Zn-rich layers and the lower Z-contrast, the $\mathrm{Mg} / \mathrm{Zn}$ layers. This result is similar to the sandwiched stacking of $\eta_{2}$, repored in our previpus work [2]. Moreover, in Fig. 4c, the FFT diffractogram, this paeticle can be recognized as $\eta_{2}{ }^{(4)}$ crystal exhibiting the crystallographic relationship with respect to the Al matrix: $[10 \overline{1} 0] \eta_{2}{ }^{(4)} / /[110]_{\mathrm{Al}}$ and $(0001) \eta_{2}{ }^{(4)} / /(\overline{1} 11)_{\mathrm{Al}}$.

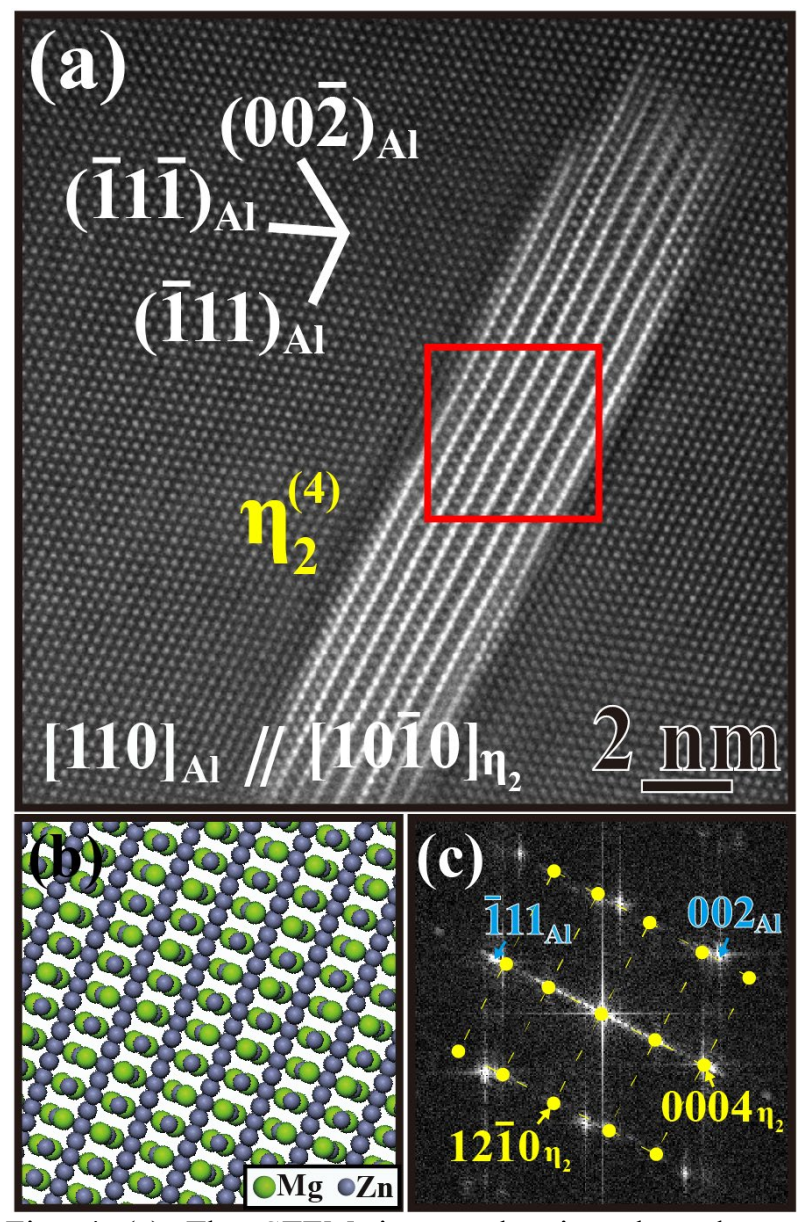

Fig. 4 (a) The STEM image showing the edge-on configuration of $\eta_{2}{ }^{(4)}$ along the [110] $]_{\mathrm{Al}}$ zone axis [13]. (b) Stimulated atomic-stacking. (c) FFT diffractogram. 
Fig. 5a shows that two edge-on configurations of two variants of $\eta_{2}$ (i.e., $\eta_{2}{ }^{(2)}$ and $\eta_{2}{ }^{(4)}$ ) exhibiting the sandwiched stacking structure on the $(0001) \eta_{2} / /(\overline{1} 1 \overline{1})_{\mathrm{A} 1}$ and $(0001) \eta_{4} / /(\overline{1} 11)_{\mathrm{Al}}$ habit planes, respectively. Their different size can be ascribed to the difference in the growth rate. Particularly, the joint area, i.e., the interweaved interface, between $\eta_{2}{ }^{(2)}$ and $\eta_{2}{ }^{(4)}$ possesses a twinning relationship with an angle of $109.5^{\circ}$ between the $(00 \overline{2})_{\mathrm{Al}}$ plane, as marked by the red lines in Fig. 5a. Here, it can be ascribed to two phenomena. First, the collision of two variants of $\eta_{2}$ occurred as they grow, the so-called hard impingement $[12,14]$. Secondly, these two $\eta_{2}$ would develop at the same interface of the nucleation stage. we temporarily call the intrinsic boundary.

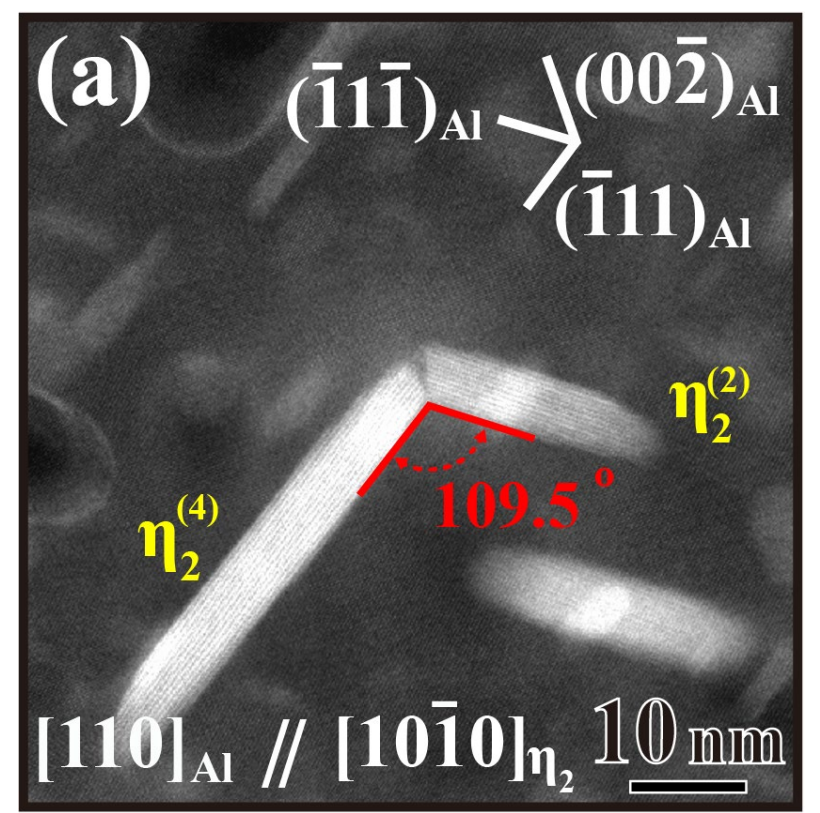

Fig. 5 (a) HAADF STEM image showing the two edgeon configurations of $\eta_{2}$ precipitate (i.e., $\eta_{2}{ }^{(2)}$ and $\eta_{2}{ }^{(4)}$ ) grown on the $(0001) \eta_{2} / /(\overline{1} 1 \overline{1})_{\mathrm{Al}}$ and $(0001) \eta_{4} / /(\overline{1} 11)_{\mathrm{Al}}$ habit planes, respectively under the $[110]_{\mathrm{Al}} / /[10 \overline{1} 0] \eta_{2}$ zone axis [13].

For the detailed investigation on the atomic arrangement and microstructural evolution in between two variants of $\eta_{2}$ (i.e., $\eta_{2}{ }^{(2)}$ and $\eta_{2}{ }^{(4)}$ ), the area with the nearly darkness Z-contrast (as marked by the red arrow in Fig 5b) can be recognized as segregation of vacancies or $\mathrm{Mg}$ solute atoms. This given area was supposedly ascribed to the early stage of nucleation. As the growth of these two variants of $\eta_{2}$, vacancies and $\mathrm{Mg}$ atoms are gradually annihilated by the substitutions of $\mathrm{Zn}$ and $\mathrm{Cu}$ solute atoms. Finally, the mismatched interface would turn into the well-matched interface (as marked by the solid dot of the red arrow in Fig. 5b). Whether the intrinsic boundary would be observed in 15 typed $\eta$ under the observation of $[110]_{\mathrm{Al}},[001]_{\mathrm{Al}}$ and $[11 \overline{2}]_{\mathrm{Al}}$ zone axes has yet to be reported.

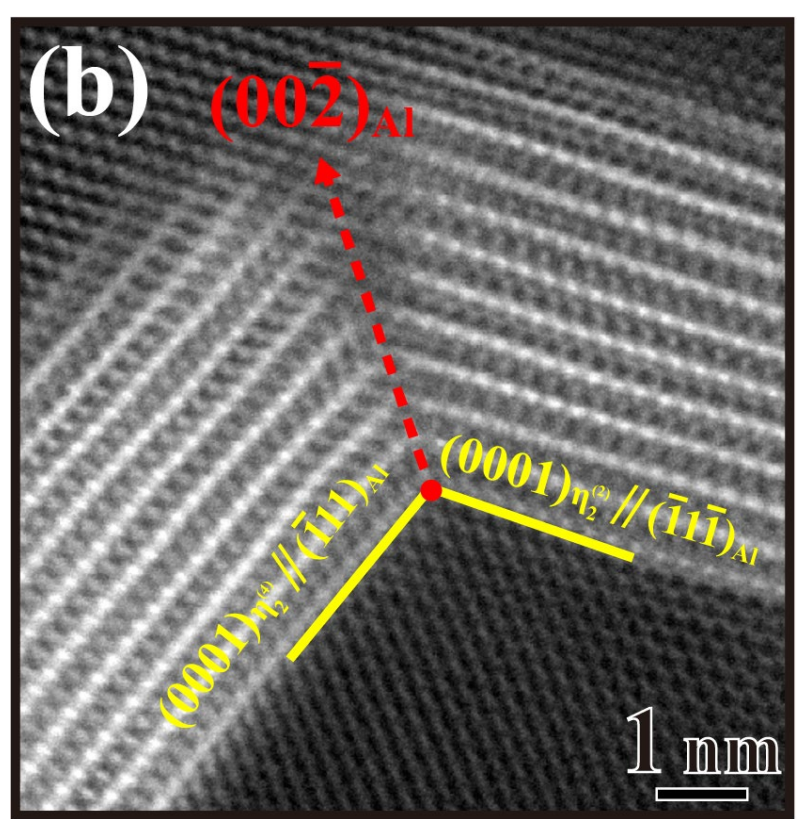

Fig. 5 (b) A enlarged image of (a) revealing the twin relationship of an angle of $109.5^{\circ}$ between the $(00 \overline{2})_{\mathrm{Al}}$

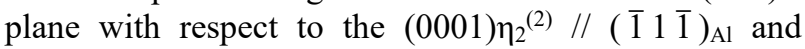
$(0001) \eta_{2}{ }^{(4)} / /(\overline{1} 11)_{\mathrm{Al}}$ habit planes [13].

Another case crystallographic relationship of different variants of $\eta_{2}$ is shown in Fig. 6a. Specially, the joint area (Fig. 6) is totally different from the end of two variants, as shown in Fig. 5. Along the zone axis of [110 $]_{\mathrm{Al}}$, the edge-on configurations of $\eta_{2}{ }^{(2)}$ and $\eta_{2}{ }^{(4)}$, grown on the $(0001) \eta_{2} / /(\overline{1} 1 \overline{1})_{\mathrm{Al}}$ and $(0001) \eta_{4} / /(\overline{1} 11)_{\mathrm{Al}}$ habit planes, respectively, reveal the twinning relationship with an angle of $109.5^{\circ}$ or $70.5^{\circ}$ with respect to two different twinning planes in between these two variants.

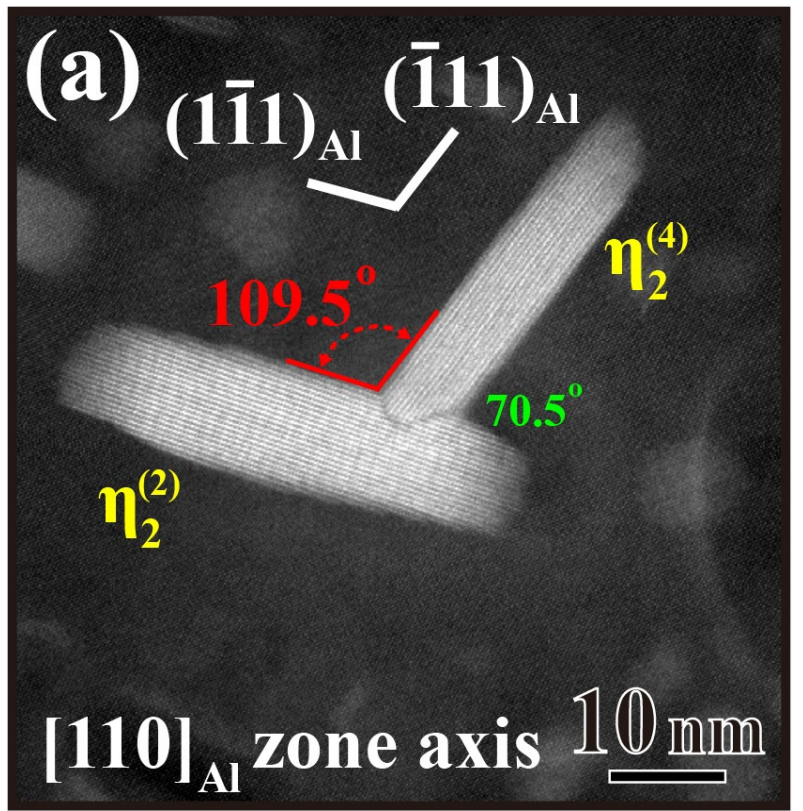

Fig. 6 (a) HAADF STEM image showing the twinning relationship with an angle of $109.5^{\circ}$ or $70.5^{\circ}$ twinning relationship with an angle of $109.5^{\circ}$ respect to two edgeon configurations of $\eta_{2}{ }^{(2)}$ and $\eta_{2}{ }^{(4)}$ along the zone axis of $[110]_{\mathrm{Al}}$. 
Similarly, the detailed study on the atomic configurations in between $\eta_{2}{ }^{(2)}$ and $\eta_{2}{ }^{(4)}$ are illustrated in Fig. 6b. It has been found that the twin relationships of two angles of $109.5^{\circ}$ and $70.5^{\circ}$ between the $(1 \overline{1} 3)_{\mathrm{Al}}$ and $(\overline{3} 31)_{\text {Al }}$ planes of the Al matrix, respectively. The coherent twin boundaries would be caused by the nucleation and growth, instead of the effect of the hard impingement. It could be suggested that the nucleation site would be the partial segregation of $\mathrm{Zn} / \mathrm{Cu}$ nearby the interfaces between the $\eta_{2}$ precipitate and the Al matrix with the abnormally strong bright Z-contrast, reported in the previous work [2]. In the present case, the nuclei would be located on the interfaces of $\eta_{2}{ }^{(2)}$ and would follow the twinning relationship as the growth of precipitates.

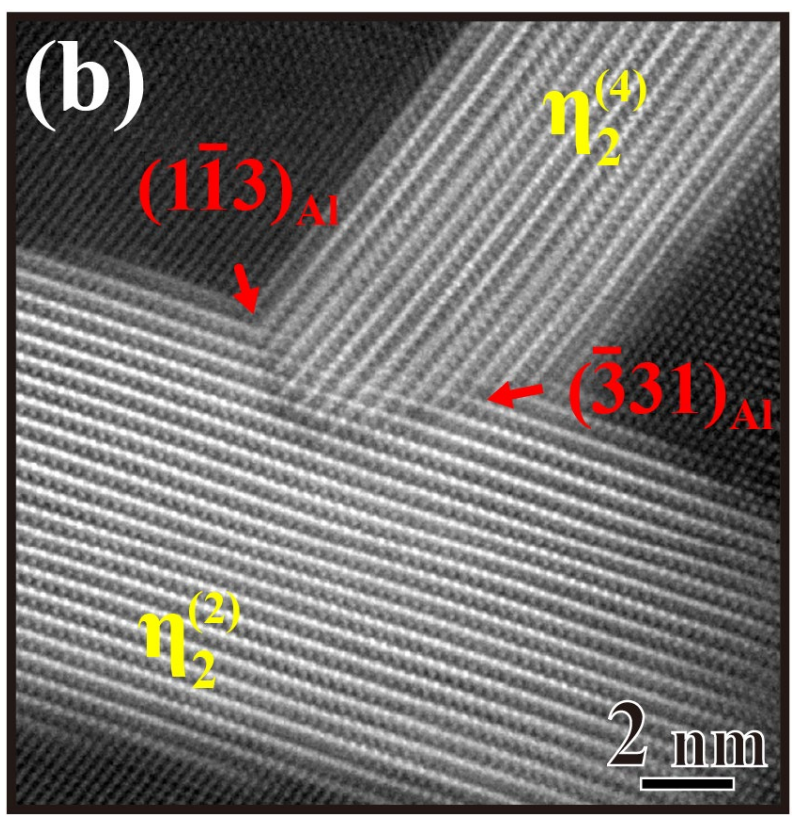

Fig. 6 (b) A enlarged image of (a) revealing the twin relationships of two angles of $109.5^{\circ}$ and $70.5^{\circ}$ between the $(1 \overline{1} 3)_{\mathrm{Al}}$ and $(\overline{3} 31)_{\mathrm{Al}}$ planes with respect to $\eta_{2}{ }^{(2)}$ and $\eta_{2}{ }^{(4)}$ precipitates.

Furthermore, in Fig. 7, the edge-on configurations of $\eta_{2}{ }^{(2)}$ and $\eta_{2}{ }^{(4)}$ are specifically observed along the zone axis of $[\overline{1} \overline{1} 0]_{\mathrm{Al}}$ with respect to the resulted HAADF STEM image of Fig. 6. At the joint or intersected position, the crystallographic relationship in twinning would exist. However, one coherent twinning boundary, as indicated by the red arrow in Fig. 7, can be found. It can be suggested that the segregations of $\mathrm{Zn} / \mathrm{Cu}$ to be the nucleation site of $\eta_{2}{ }^{(4)}$ would locate on the part of the $\eta_{2}{ }^{(2)} / \mathrm{Al}$ interfaces.

Conclusively, according to the orientation relationships of 15 typed $\eta$ precipitates $\left(\eta_{1}-\eta_{14}\right.$, and $\left.\eta_{4}{ }^{\prime}\right)$ and the corresponding variants, their crystallographic relationships are related to be twinning. In the present work, along the zone axes of $[110]_{\mathrm{Al}}$ and $[\overline{1} \overline{1} 0]_{\mathrm{Al}}$, it can be fount the twinning boundaries in between the edge-on configurations of $\eta_{2}^{(2)}$ and $\eta_{2}{ }^{(4)}$.

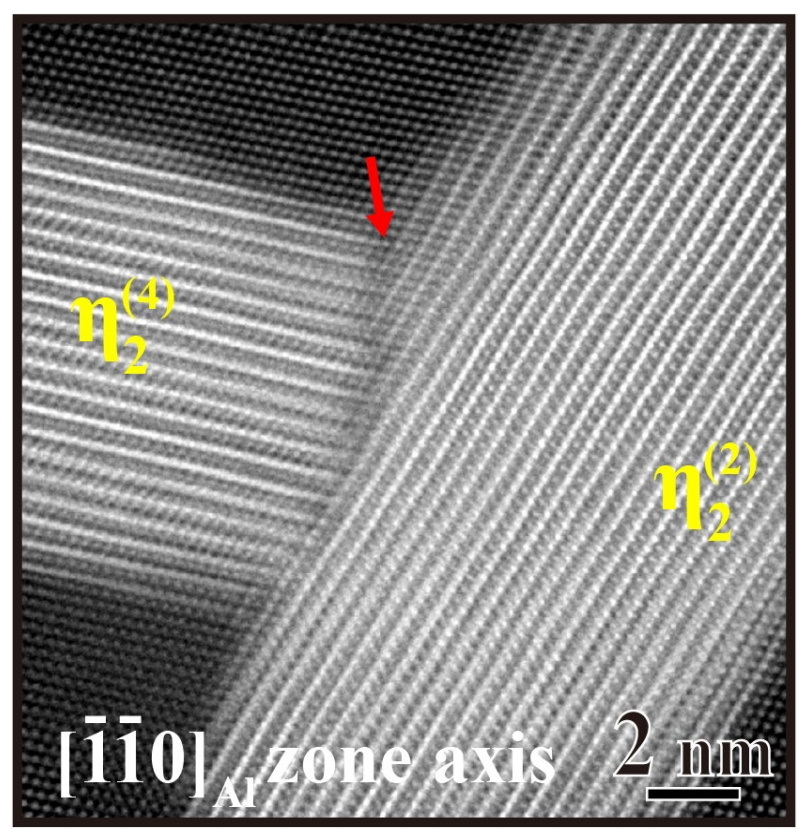

Fig. 7 HAADF STEM image along the zone axis of $[110]_{\mathrm{Al}}$ with respect to that of Fig. 6 revealing the atomic configuration of the joint area in between $\eta_{2}{ }^{(2)}$ and $\eta_{2}{ }^{(4)}$.

\section{Acknowledgements}

This work was carried out with the financial support from Foundation for the Advancement of Outstanding Scholarship and Ministry of Science and Technology (Taiwan) under Contract MOST108-2622-E-002-016-CC2. The authors are grateful to Taiwan Instrument Research Institute and Materials Analysis Technology Inc. (MA-tek) for technical advice.

\section{Reference}

[1] T.-F. Chung, Y.-L. Yang, B.-M. Huang, Z. Shi, J. Lin, T. Ohmura, J.-R. Yang, Acta Mater. 149 (2018).

[2] T.-F. Chung, Y.-L. Yang, M. Shiojiri, C.-N. Hsiao, W.-C. Li, C.-S. Tsao, Z. Shi, J. Lin, J.-R. Yang, Acta Mater. 174 (2019).

[3] A. Deschamps, A. Bigot, F. Livet, P. Auger, Y. Brechet, D. Blavette, Philos. Mag. A 81 (2001).

[4] A. Deschamps, F. Bley, F. Livet, D. Fabregue, L. David, Philosophical Magazine 83 (2003).

[5] L. Couturier, A. Deschamps, F. De Geuser, F. Fazeli, W.J. Poole, Scripta Mater. 136 (2017).

[6] C.R. Hutchinson, F. de Geuser, Y. Chen, A. Deschamps, Acta Mater. 74 (2014).

[7] A. Bendo, K. Matsuda, A. Lervik, T. Tsuru, K. Nishimura, N. Nunomura, R. Holmestad, C.D. Marioara, K. Shimizu, H. Toda, Mater. Charact. (2019).

[8] H. Degischer, W. Lacom, A. Zahra, C. Zahra, Z. Metallk. 71 (1980).

[9] J. Gjønnes, C.J. Simensen, Acta. Metall. Mater. 18 (1970).

[10] S. Ringer, K. Hono, Mater. Charact. 44 (2000).

[11] J. da Costa Teixeira, D.G. Cram, L. Bourgeois, T.J. Bastow, A.J. Hill, C.R. Hutchinson, Acta Mater. 56 (2008).

[12] T.-F. Chung, Y.-L. Yang, C.-N. Hsiao, W.-C. Li, B.M. Huang, C.-S. Tsao, Z. Shi, J. Lin, P.E. Fischione, T. 
Ohmura, J.-R. Yang, International Journal of Lightweight Materials and Manufacture 1 (2018).

[13] T.-F. Chung, Y.-L. Yang, M. Shiojiri, C.-N. Hsiao, W.-C. Li, J.-R. Yang, Procedia Manufacturing 37 (2019).

[14] B. Decreus, A. Deschamps, F. De Geuser, P. Donnadieu, C. Sigli, M. Weyland, Acta Mater. 61 (2013). 